\title{
Insomnia and Susceptibility to Depressive Symptoms and Fatigue in Diverse Breast Cancer Survivors
}

\author{
Reina Haque, PhD, MPH, ${ }^{1,2}$ Jin Wen Hsu, PhD, ${ }^{1}$ Chantal Avila, MA, ${ }^{1}$ Richard Olmstead, PhD, ${ }^{3}$ \\ Judith E. Carroll, $\mathrm{PhD},{ }^{3}$ and Michael R. Irwin, $\mathrm{MD}, \mathrm{PhD}^{3}$
}

\begin{abstract}
Background: Nearly $40 \%$ of breast cancer survivors have insomnia, yet, information how this condition affects their quality of life is lacking. We examined the association between insomnia and depressive symptoms and fatigue in breast cancer survivors.

Methods: Participants were recruited from a community health plan. We conducted a cross-sectional analysis to examine the association between current insomnia (using Insomnia Severity Index [ISI]) and current depressive symptoms (using Inventory of Depressive Symptomology [IDS]) and fatigue (using Fatigue Symptom Inventory [FSI]) in 315 breast cancer survivors who did not have major depressive disorder. We calculated odds ratios (ORs) and 95\% confidence intervals (CIs) using multivariable logistic regression.

Results: The cohort included 30\% minority women whose median time since breast cancer diagnosis was 6 years. Survivors with current insomnia symptoms (ISI $\geq 8$ ) had a sixfold greater odds of current depressive symptoms (IDS $>14, \mathrm{OR}=5.98,95 \% \mathrm{CI}$ : 3.04-11.76), after adjusting for lifetime insomnia history $(\mathrm{OR}=2.01$, 95\% CI: $1.03-3.94)$ and perceived stress $(\mathrm{OR}=6.37,95 \% \mathrm{CI}: 2.48-16.32)$. Insomnia symptoms were markedly associated with moderate fatigue (FSI $>3, \mathrm{OR}=5.02,95 \% \mathrm{CI}$ : 2.66-9.44). Ever use of antidepressants or sleep medications post-breast cancer diagnosis was not associated with lower odds of current depressive symptoms or feeling fatigued in those with insomnia symptoms.

Conclusion: Current insomnia symptoms were strongly correlated with current depressive symptoms and fatigue. Survivorship care plans should consider incorporating insomnia screening to that may potentially enhance quality of life domains.
\end{abstract}

Keywords: breast cancer, depressive symptoms, depression, fatigue, insomnia, quality of life

\section{Introduction}

$\mathbf{F}$ OR MANY OF THE 3.8 million female breast cancer survivors living in the United States in 2019, improved survival is complicated by long-term psychosocial effects including sleep problems, depressive symptoms, and fatigue. ${ }^{1-18}$ The prevalence of insomnia symptoms is nearly $40 \%$ in cancer survivors versus $10 \%-15 \%$ in the general population. ${ }^{19}$ Likewise, depressive symptoms and chronic fatigue in breast cancer survivors are nearly three to five times greater than in the community. ${ }^{4,11,16-18}$ Recent studies suggest that insomnia, fatigue, and depressive symptoms affect relationship distress, increased cancer-related pain, and decreased work productivity. ${ }^{20,21}$ Studies also suggest a link between cancer mortality and depression. ${ }^{22,23}$ Breast cancer survivors are more likely to have physical symptoms (fatigue, lymphedema, and weight gain) than other cancer survivors possibly due to treatment side effects. ${ }^{24,25}$

Sparse population-based data exist about the contribution of insomnia symptoms to occurrence of depressive symptoms in breast cancer survivors, and whether these conditions are modified by history of depression or lifetime insomnia history ${ }^{13-15}$ or other patient and clinical factors. Hence, it is unknown if insomnia symptoms, psychosocial history, physical health status, race/ethnicity, prior cancer treatments, or if a combination of these factors are related to depressive

\footnotetext{
${ }^{1}$ Department of Research \& Evaluation, Kaiser Permanente Southern California, Pasadena, California, USA.

${ }^{2}$ Department of Health Systems Science, Kaiser Permanente Bernard J. Tyson School of Medicine, Pasadena, California, USA.

${ }^{3}$ Cousins Center for Psychoneuroimmunology, UCLA Semel Institute for Neuroscience and Department of Psychiatry and Biobehavioral Sciences, David Geffen School of Medicine, University of California, Los Angeles, Los Angeles, California, USA.

(C) Reina Haque et al. 2020; Published by Mary Ann Liebert, Inc. This Open Access article is distributed under the terms of the Creative Commons Attribution Noncommercial License (http://creativecommons.org/licenses/by-nc/4.0/) which permits any noncommercial use, distribution, and reproduction in any medium, provided the original author(s) and the source are cited.
} 
symptoms in breast cancer survivors who are not currently comorbid for a major depression. Breast cancer survivors also suffer from persistent fatigue. ${ }^{16-18}$ Breast cancer status or its treatments can affect physical functioning long after completion of primary cancer treatments. Thus, our aim was to examine the correlation of insomnia symptoms (independent variable) with occurrence of depressive symptoms and fatigue (main outcomes) $)^{21,23}$ in breast cancer survivors, accounting for covariates such as demographics, ${ }^{26}$ tumor characteristics, ${ }^{27}$ cancer treatments, ${ }^{26-32}$ comorbidities at cancer diagnosis, ${ }^{33}$ physical activity, ${ }^{26}$ psychosocial history, ${ }^{34-36}$ and use of antidepressants or sleep medications. We evaluated these specific covariates because they have been linked with both depressive symptoms and fatigue in cancer survivors in prior studies. ${ }^{20-36}$ This knowledge can inform clinical management of long-term breast cancer survivors to improve quality of life.

\section{Methods}

\section{Study design and setting}

We conducted a cross-sectional analysis nested within a prospective cohort study. In the parent cohort study, we enrolled female breast cancer patients from Kaiser Permanente Southern California (KPSC), an integrated health care delivery system that comprised 15 hospitals and 4.7 million members. The follow-up visits of the parent prospective cohort study after the baseline interview occurred at 8-month intervals up to 32 months. The parent study's aim was to assess the role of inflammatory serum biomarkers and insomnia on the risk of developing new depression episodes. Cross-sectional results from the baseline interview are presented here. The study was approved by Internal Review Boards (IRBs) of KPSC and University of California, Los Angeles (UCLA); we obtained written informed consent from all women.

\section{Participants and data sources}

Inclusion criteria. We identified participants from the KPSC-SEER (Surveillance Endpoints and End Results)affiliated cancer registry. Women were included if they were between 55 and 85 years of age at breast cancer diagnosis, postmenopausal, had early stage (AJCC TNM Stages 0-II) breast cancer diagnosed between January 1996 and December 2012, at least 2 years postcancer treatment, with no cancer recurrence, and lived in Los Angeles county (Fig. 1, $N=3222$ ). Inclusion of postmenopausal women only was important to reduce variability in sleep measures that occur as a side effect of hormonal differences among pre-, peri-, and postmenopausal women.

Exclusion criteria. Given the parent study's aims, we excluded women who had conditions that indicated chronic inflammation (lymphedema, recent myocardial infarction, rheumatoid arthritis, body mass index [BMI] $>35 \mathrm{~kg} / \mathrm{m}^{2}$, diabetes, or on immunosuppressive therapy) or had sleep apnea or unremitted depression in the last 3 months before the baseline interview. Women were screened for eligibility in two stages: prescreening based on patients' electronic medical record (EMR) review and via phone-screening for conditions that were not captured in the EMR. A spreadsheet of all the exclusion factors was designed for the EMR review and phone-screening. For the EMR review, research associ- ates reviewed the problem list of each participant to determine the presence or absence of the aforementioned exclusion conditions. Thus, we prescreened women via EMR and specifically excluded the following women $(N=1035)$ : (1) not a current KPSC member in past 12 months; (2) nonEnglish speaking; (3) current antipsychotic prescription in last 3 months; (4) current corticosteroids or other immunosuppressive therapy last 3 months; (5) diagnosed with lymphedema (swelling that increases nocturnal pain), recent $(<1$ year) myocardial infarction, rheumatoid arthritis, BMI $>35 \mathrm{~kg} / \mathrm{m}^{2}$, or diabetes since breast cancer diagnosis; or (6) not ambulatory. This left 2187 total women eligible for phone screening for additional exclusion factors. Women were asked if they received a diagnosis of the following exclusion conditions from a health care provider in the last 3 months: sleep apnea, any psychiatric disorder, or current major depression.

We then obtained assent from the participants' primary care providers via electronic mail to ensure that their patients did not recently die; all providers assented. We then sent invitation letters to potential participants, along with response cards to accept or decline. Of these 2187 eligible women, $n=1072$ refused $(49.0 \%), n=635(29.0 \%)$ could not be contacted by phone, and $n=109(4.9 \%)$ were deemed ineligible based on the phone-screening of the additional aforementioned exclusion factors. Thus, total of 371 women completed the phone screening (response fraction $=371$ / $[2187-635-109]=25.7 \%$ ) and met the eligibility criteria for participation; of these, 56 refused, leaving 315 (315/ $371=84.9 \%$ ) eligible women who enrolled in the study. The baseline interviews were conducted between August 2013 and March 2015.

\section{Main outcomes}

Data elements were captured from three sources: (1) inperson interviews administered by trained masters' level research associates in a private office at the UCLA medical center (research associates were supervised by the study clinician (M.R.I.), and they ascertained demographics, vasomotor symptoms, lifetime depression history based on the Structured Clinical Interview for DSM-IV (SCID-IV)), lifetime insomnia history based on the Structured Interview for Sleep Disorders (SIS-D). (2) A questionnaire booklet given to participants that was completed on the same interview day included the Inventory of Depressive Symptomatology, SelfReport (IDS-SR); Fatigue Symptom Inventory (FSI); Insomnia Severity Index (ISI); Perceived Stress Scale (PSS); Risky Families Questionnaire (RFQ); and Godin Leisure Physical Activity Scale (GLPAS). (3) EMR (clinical and cancer treatment variables were extracted by the biostatistician).

The appointment for interviewer-led and self-reported booklet questionnaire took 1 hour combined; all data were entered into an Access database by the research associates. To assess psychosocial outcomes, we used validated questionnaires described below.

Depressive symptoms. The IDS-SR ascertained symptoms in the last 7 days (Cronbach's $\alpha=0.88$ ). The 30-item validated IDS-SR was based on DSM-IV criteria for major depressive disorder and scored according to published protocol. ${ }^{37,38}$ Higher scores indicated greater severity of depressive symptoms; this variable was dichotomized to none 

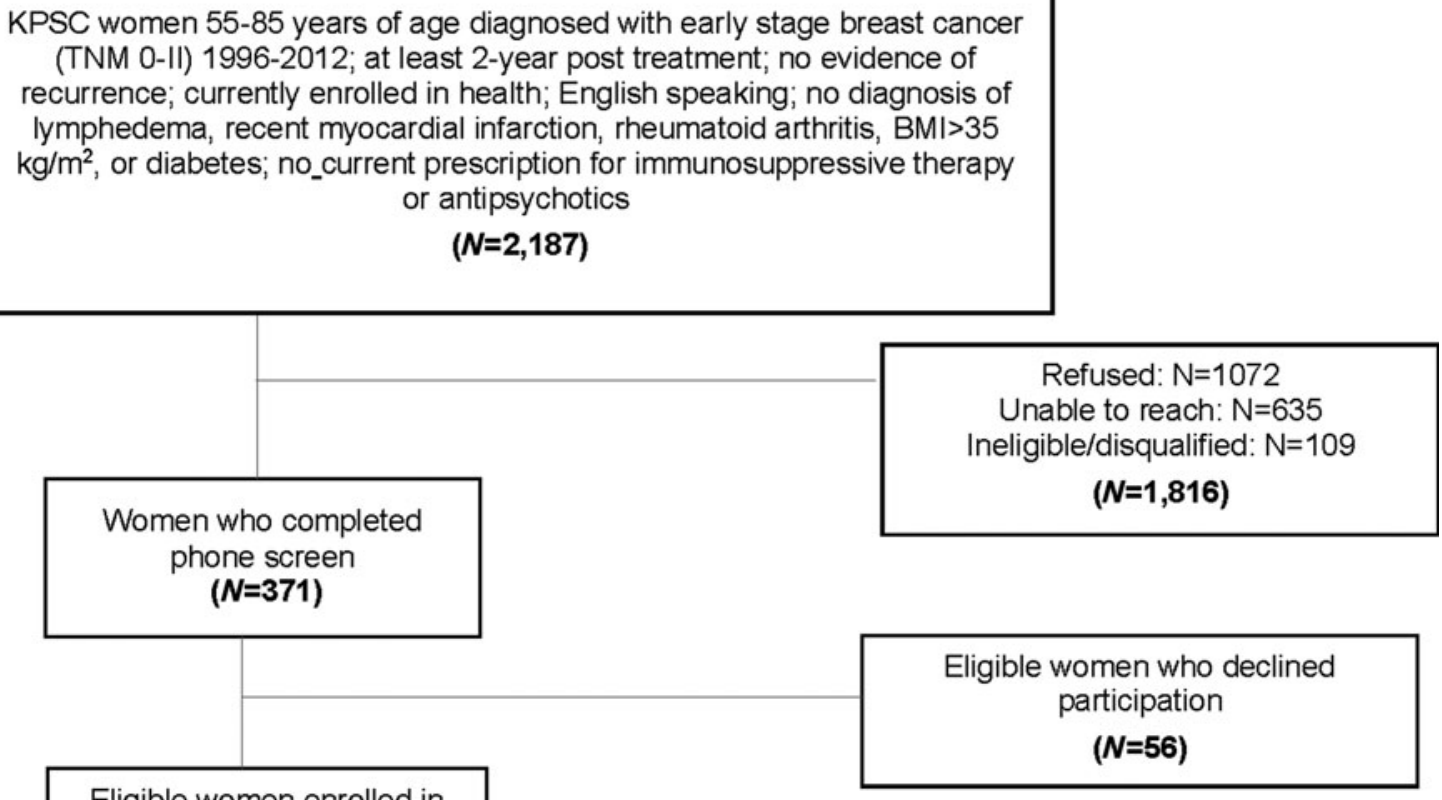

FIG. 1. Study cohort of breast cancer survivors.

$(<14)$ and some (mild, moderate, and severe, $\geq 14)$. In the analysis, we examined IDS-SR scores after removing responses items on sleep.

Fatigue. We used the FSI, a validated 14-item questionnaire developed for patients with cancer (Cronbach's $\alpha=0.94) .{ }^{39}$ Questions that assessed average fatigue, most fatigue, and least fatigue in the past week were averaged to obtain a measure of severity. The FSI score was dichotomized into none (FSI $<3$ ) and some (FSI $\geq 3$ indicated mild, moderate, and severe fatigue) based on prior literature. ${ }^{39}$

\section{Main independent variable}

Insomnia symptoms. The validated ISI is a 7-question self-reported questionnaire (Cronbach's $\alpha=0.88$ ) that assessed both nighttime and daytime insomnia symptoms in the last 2 weeks. ${ }^{40}$ The questionnaire sums to a total score ranging from 0 to 28 . The following totals indicated absence of insomnia (0-7); subthreshold insomnia (8-14); moderate insomnia (15-21); and severe insomnia (22-28). ${ }^{41}$ In our analysis, we used ISI $>8$ to indicate current subthreshold to severe insomnia and subsequently use the term "insomnia symptoms." This cutoff was also used because in our analysis of ISI data, very few women reported moderate $(N=23)$ or severe $(N=3)$ insomnia, thus to avoid error in analyses of groups with inadequate sample size, we selected an ISI $>8$ to indicate "insomnia symptoms."

\section{Covariates: other psychosocial histories}

Lifetime depression history. At baseline, interviewers administered the SCID-IV to obtain lifetime history of depression. $^{37,38}$
Lifetime insomnia history. To obtain lifetime history of insomnia, we used the Insomnia Evaluation Interview (from the validated SIS-D ${ }^{42}$ ). Lifetime insomnia history was identified if participants had ever been bothered by insomnia for more than 1 month.

Perceived Stress Scale-14. This 14-item questionnaire was administered to ascertain women's perception of how stressful their lives were in the past month (Cronbach's $\alpha=0.82)$. ${ }^{40}$ Scores were totaled; we defined PSS $>14$ to indicate having some stress. ${ }^{40}$

Risky Families Questionnaire. To assess the degree of physical and emotional abuse, neglect, and lack of affection during childhood and adolescence, we used the RFQ ${ }^{43,44}$ with seven questions with a score range of $7-28$, with higher scores reflecting greater early stress (Cronbach's $\alpha=0.89$ ).

\section{Covariates: clinical, and demographic variables}

Demographic characteristics. We captured the following current demographic and clinical covariates from the interview: employment status, living arrangements, physical activity $\left(\mathrm{GLPAS}^{45}\right)$, education, and race/ethnicity at the baseline interview. At baseline, we captured vasomotor symptoms (current hot flashes/night sweats) occurring in the last 3 months ("During the past three months, did hot flashes or night sweats interfere with your sleep?"'). ${ }^{46}$ Women were also queried about their history of use of antidepressants or sleep aids (prescriptions or over the counter) anytime postbreast cancer diagnosis (ever/never) up to the baseline interview date. Thus, the variable representing "ever" antidepressant and sleep aid use were proxies of past use, but it could reflect recent use for some fraction of women as well. 
Clinical variables. Age and year of breast cancer diagnosis, stage, and receipt of adjuvant therapies (radiation, chemotherapy, and hormonal therapy) were obtained from the KPSC-(SEER)-affiliated cancer registry. Charlson comorbidity index was calculated using the Elixhauser methods-based EMR data. ${ }^{47}$

\section{Statistical analysis}

Descriptive statistics provided frequencies and proportions for categorical variables, including the frequency distribution of the main outcomes, current depressive symptoms (ascertained IDS) and current fatigue (assessed by FSI) by current insomnia symptoms (i.e., ISI >8). We examined the covariates, lifetime depression history, and lifetime insomnia history, as potential confounders of the association between insomnia symptoms (independent variable) and depressive symptoms and fatigue (study outcomes). We calculated odds ratios (ORs) and 95\% confidence intervals (CIs) for the association of these outcomes (current depressive symptoms and current fatigue) by insomnia symptoms, accounting for lifetime depression and lifetime insomnia histories using logistic regression. In addition, we calculated the ORs and 95\% CI for the association of these outcomes with each of the demographic, clinical and tumor characteristics.

We conducted multivariable logistic models to calculate the adjusted ORs and 95\% CIs for the association between current insomnia symptoms and current depressive symptoms (IDS; or current fatigue, FSI) accounting for age at interview, stage at breast cancer diagnosis, years since breast cancer diagnosis, race/ethnicity, ever antidepressant use, ever sleep medication use, current perceived stress, early childhood stress, lifetime depression history, and lifetime insomnia history (because these variables were deemed important given clinical relevance and our bivariate analyses). The psychosocial covariates were not highly correlated based on our check of the multicollinearity using the Spearman correlation (range: 0.06-0.42). All statistical analyses were performed using the software program SAS 9.4 (SAS Institute, Inc.).

\section{Results}

\section{Demographic and clinical characteristics}

In the cohort of 315 breast cancer survivors, the mean age at interview was 70.8 years (standard deviation [SD] 6.4) and mean years since breast cancer diagnosis was 6.3 years (SD 3.9, range: 2.0-17.0). The distribution of early stage breast cancer was as follows: stage 0 (ductal carcinoma in situ): $19.4 \%$; stage I: $47.6 \%$, and stage II: $33.0 \%$. The diverse cohort included $70.2 \%$ white women (the $n=4$ Hispanic women were included with the white group); $20.1 \%$ African Americans, and 8.9\% Asian women. Over half (60.8\%) were college educated.

Nearly $72.4 \%$ had a Charlson comorbidity index score $>1$, while $27.6 \%$ had no other comorbidity (Table 1 ). The comorbidities mainly included hypertension $(60.0 \%)$, chronic obstructive pulmonary disease $(15.6 \%)$, and renal disease (16.8\%). Prevalence of vasomotor symptoms (hot flashes/ night sweats) was $55.6 \%$. Nearly $30.4 \%$ reported at least one episode of insomnia in their lifetime, while $33.0 \%$ reported having a lifetime depressive episode. Prevalence of ever using sleep medications (prescription or over the counter) any time after breast cancer diagnosis was $16.5 \%$. Almost $17.1 \%$ of women reported ever using antidepressants after their initial breast cancer diagnosis. Among those who used antidepressants, the reasons varied: management of depression $(55.6 \%)$, hot flashes $(24.1 \%)$, sleeping problems $(25.9 \%)$, or other problems (25.9\%; not mutually exclusive; data not shown). As expected for breast cancer survivors, use of hormonal therapies (tamoxifen or aromatase inhibitors) was common (38.7\%).

\section{Breast cancer treatments, tumor characteristics, and demographics}

Regarding time since breast cancer diagnosis, we found no differences in the odds of current depressive symptoms when comparing recent survivors ( $<5$ years) to long-term survivors ( $\geq 5$ years) Tables 3 and 4 . Similarly, we found no differences in the odds of current depressive symptoms or current fatigue by the stage of breast cancer, nor by type of adjuvant therapy (chemotherapy, radiation, or hormonal), or current physical activity, although we noted a signal that those who reported being active or moderately active were less likely to report current insomnia symptoms (Table 1).

\section{Correlates of current insomnia symptoms}

Overall, 104 (33\%) breast cancer survivors reported having insomnia symptoms (ISI $>8$ ) (Table 1); the distribution was subthreshold $(N=78)$; moderate $(N=23)$; and severe $(N=3)$, and therefore, we dichotomized this variable. Factors strongly correlated with current insomnia symptoms were current perceived stress $(\mathrm{OR}=3.96,95 \% \mathrm{CI}: 2.08-7.57)$ and lifetime insomnia history $(\mathrm{OR}=6.63,95 \% \mathrm{CI}$ : $3.91-11.25)$, but not lifetime history of depression $(\mathrm{OR}=1.63,95 \% \mathrm{CI}$ : 1.00-2.66). Current insomnia symptoms were fourfold greater in those with current perceived stress (PSS $>14$; $\mathrm{OR}=3.96,95 \%$ CI: 2.08-7.57), and in those had experienced more early life stress $(p=0.05)$. The odds of current insomnia symptoms was threefold greater in women who ever used sleep medications (overall $\mathrm{OR}=3.16,95 \% \mathrm{CI}$ : $1.72-5.81$ ).

The odds of insomnia symptoms were also elevated in those who ever used antidepressants, but the association did not reach statistical significance $(\mathrm{OR}=1.36,95 \% \mathrm{CI}$ : 0.74 2.50). The associations between insomnia symptoms and antidepressants and sleep medications are expected given that more patients with this condition used such medications.

\section{Insomnia symptoms (ISI >8) and depressive symptoms $(I D S>14)$}

The prevalence of current depressive symptoms (IDS >14 score) at baseline was 29\% (Table 2). In bivariate analyses, the following factors were strongly correlated with current depressive symptoms: current insomnia symptoms $(\mathrm{OR}=7.63$, 95\% CI: 4.44-13.10); current perceived stress $(\mathrm{OR}=8.71,95 \% \mathrm{CI}: 3.64)$; lifetime insomnia history $(\mathrm{OR}=$ 3.98, 95\% CI: 2.37-6.68); and ever use of antidepressant medications $(\mathrm{OR}=2.50,95 \% \mathrm{CI}: 1.37-4.56)$.

These strong associations persisted in the multivariable models, with the odds of current depressive symptoms being sixfold greater in those who reported current insomnia symptoms (adjusted OR $=5.98,95 \%$ CI: 3.04-11.76) after adjusting for age, breast cancer stage, years since cancer 
Table 1. Demographics, Clinical, and Tumor Characteristics by Baseline Insomnia Severity Index

\begin{tabular}{|c|c|c|c|c|}
\hline Total & $\begin{array}{c}\text { None } \\
(I S I<8 \text { or unknown }) \\
\mathrm{N}=211, \mathrm{~N}(\%)^{\mathrm{a}}\end{array}$ & $\begin{array}{c}\text { Subthreshold- } \\
\text { severe }(I S I \geq 8) \text {, } \\
\mathrm{N}=104, \mathrm{~N}(\%)^{\mathrm{a}}\end{array}$ & $\begin{array}{l}\text { Total, } \\
\mathrm{N}(\%)^{\mathrm{a}}\end{array}$ & $\begin{array}{c}\text { Overall } \\
\text { OR }(95 \% \text { CI })\end{array}$ \\
\hline \multicolumn{5}{|l|}{ Age at interview (years) } \\
\hline$\leq 65$ & $38(18.01)$ & $21(20.19)$ & $59(18.73)$ & Ref. \\
\hline$>65$ & $173(81.99)$ & $83(79.81)$ & $256(81.27)$ & $0.87(0.48-1.57)$ \\
\hline \multicolumn{5}{|l|}{ Race/ethnicity } \\
\hline White (Hispanic and non-Hispanic) & $143(67.77)$ & $78(75.00)$ & $221(70.16)$ & Ref. \\
\hline African American & $46(21.80)$ & $20(19.23)$ & $66(20.95)$ & $0.80(0.44-1.44)$ \\
\hline Asian & $22(10.43)$ & $6(5.77)$ & $28(8.89)$ & $0.50(0.19-1.29)$ \\
\hline \multicolumn{5}{|l|}{ Stage at $\mathrm{BC} d x$} \\
\hline Stage 0 (DCIS) & $49(23.22)$ & $12(11.54)$ & $61(19.37)$ & Ref. \\
\hline Stages I-II & $162(76.78)$ & $92(88.46)$ & $254(80.63)$ & $2.32(1.17-4.58)$ \\
\hline \multicolumn{5}{|l|}{ Years since diagnosis } \\
\hline$\leq 5$ & $106(50.24)$ & $51(49.04)$ & $157(49.84)$ & Ref. \\
\hline$>5$ & $105(49.76)$ & $53(50.96)$ & $158(50.16)$ & $1.05(0.66-1.68)$ \\
\hline \multicolumn{5}{|l|}{ Radiation } \\
\hline No & $101(47.87)$ & $48(46.15)$ & $149(47.30)$ & Ref. \\
\hline Yes & $110(52.13)$ & $56(53.85)$ & $166(52.70)$ & $1.07(0.67-1.72)$ \\
\hline \multicolumn{5}{|l|}{ Hormonal (Tamoxifen/AIs) } \\
\hline No & $108(51.18)$ & $46(44.23)$ & $154(48.89)$ & Ref. \\
\hline Yes & $103(48.82)$ & $58(55.77)$ & $161(51.11)$ & $1.32(0.82-2.12)$ \\
\hline \multicolumn{5}{|l|}{ Chemotherapy } \\
\hline No & $156(73.93)$ & $66(63.46)$ & $222(70.48)$ & Ref. \\
\hline Yes & $55(26.07)$ & $38(36.54)$ & $93(29.52)$ & $1.63(0.99-2.70)$ \\
\hline \multicolumn{5}{|l|}{ Charlson Comorbidity Index } \\
\hline 0 & $61(28.91)$ & $26(25.00)$ & $87(27.62)$ & Ref. \\
\hline $1-2$ & $78(36.97)$ & $47(45.19)$ & $125(39.68)$ & $1.41(0.79-2.54)$ \\
\hline $3+$ & $72(34.12)$ & $31(29.81)$ & $103(32.70)$ & $1.01(0.54-1.88)$ \\
\hline \multicolumn{5}{|l|}{ Physical activity } \\
\hline Insufficiently active & $47(24.74)$ & $25(25.00)$ & $72(24.83)$ & Ref. \\
\hline Moderately active & $48(25.26)$ & $29(29.00)$ & $77(26.55)$ & $1.14(0.58-2.22)$ \\
\hline Active & $95(50.00)$ & $46(46.00)$ & $141(48.62)$ & $0.91(0.50-1.66)$ \\
\hline Unknown/missing & $21(\mathrm{~N} / \mathrm{A})$ & 4 (N/A) & 25 (N/A) & N/A \\
\hline \multicolumn{5}{|l|}{ RFQ } \\
\hline Mean & 1.72 & 1.92 & 1.79 & \\
\hline Median & 1.36 & 1.59 & 1.45 & $p=0.052$ \\
\hline Lower, upper quartiles & $1.18-2.00$ & $1.18-2.36$ & $1.18-2.18$ & \\
\hline \multicolumn{5}{|l|}{ PSS } \\
\hline None $(0-13)$ & $75(36.41)$ & $13(12.62)$ & $88(28.48)$ & Ref. \\
\hline Some $(14+)$ & $131(63.59)$ & $90(87.38)$ & $221(71.52)$ & $3.96(2.08-7.57)$ \\
\hline Unknown/missing & 5 (N/A) & 1 (N/A) & $6(\mathrm{~N} / \mathrm{A})$ & \\
\hline \multicolumn{5}{|l|}{ Vasomotor symptoms } \\
\hline No & 97 (47.32) & $39(38.61)$ & $136(44.44)$ & Ref. \\
\hline Yes & $108(52.68)$ & $62(61.39)$ & $170(55.56)$ & $1.43(0.88-2.32)$ \\
\hline Unknown/missing & 6 (N/A) & 3 (N/A) & 9 (N/A) & N/A \\
\hline \multicolumn{5}{|l|}{ Lifetime depression history ${ }^{\mathrm{b}}$} \\
\hline No & $149(70.62)$ & $62(59.62)$ & $211(66.98)$ & Ref. \\
\hline Yes & $62(29.38)$ & $42(40.38)$ & $104(33.02)$ & $1.63(1.00-2.66)$ \\
\hline \multicolumn{5}{|l|}{ Lifetime insomnia history } \\
\hline No & $175(82.94)$ & $44(42.31)$ & $219(69.52)$ & Ref. \\
\hline Yes & $36(17.06)$ & $60(57.69)$ & $96(30.48)$ & $6.63(3.91-11.25)$ \\
\hline \multicolumn{5}{|l|}{ Antidepressants (use post-BC dx) } \\
\hline No & $178(84.36)$ & $83(79.81)$ & $261(82.86)$ & Ref. \\
\hline Yes & 33 (15.64) & $21(20.19)$ & $54(17.14)$ & $1.36(0.74-2.50)$ \\
\hline \multicolumn{5}{|l|}{ Sleep medication (use post-BC dx) } \\
\hline No & $188(89.10)$ & $75(72.12)$ & $263(83.49)$ & Ref. \\
\hline Yes & $23(10.90)$ & $29(27.88)$ & $52(16.51)$ & $3.16(1.72-5.81)$ \\
\hline
\end{tabular}

${ }^{\mathrm{a}} p$-Values and percents are based on known values.

${ }^{\mathrm{b}}$ Lifetime depression history= determined from baseline DSM IV/V SCID interview.

95\% CI, 95\% confidence interval; BC, breast cancer; ISI, Insomnia Severity Index; OR, odds ratio; PSS, Perceived Stress Scale; RFQ,

Risky Families Questionnaire; SCID, Structured Clinical Interview for DSM.

DCIS, ductal carcinoma in situ. 
Table 2. Demographics, Clinical, and Tumor Characteristics by Baseline Inventory of Depressive SyMPTOMOLOGY

\begin{tabular}{|c|c|c|c|c|}
\hline Total & $\begin{array}{c}\text { None } \\
\text { (IDS <14 or unknown), } \\
\mathrm{N}=223, \mathrm{~N}(\%)\end{array}$ & $\begin{array}{c}\text { Some }(I D S \geq 14) \\
\mathrm{N}=92, \mathrm{~N}(\%)\end{array}$ & Total, $\mathrm{N}(\%)$ & $\begin{array}{c}\text { Overall OR } \\
(95 \% C I)\end{array}$ \\
\hline \multicolumn{5}{|l|}{ Age at interview (years) } \\
\hline$\leq 65$ & $41(18.39)$ & $18(19.57)$ & 59 (18.73) & Ref. \\
\hline$>65$ & $182(81.61)$ & $74(80.43)$ & $256(81.27)$ & $0.93(0.50-1.72)$ \\
\hline \multicolumn{5}{|l|}{ Race/ethnicity } \\
\hline White (Hispanic and non-Hispanic) & $158(70.85)$ & $63(68.48)$ & $221(70.16)$ & Ref. \\
\hline African American & 45 (20.18) & $21(22.83)$ & $66(20.95)$ & $1.17(0.65-2.12)$ \\
\hline Asian & $20(8.97)$ & $8(8.70)$ & $28(8.89)$ & $1.00(0.42-2.40)$ \\
\hline \multicolumn{5}{|l|}{ Stage at BC dx } \\
\hline Stage 0 (DCIS) & 49 (21.97) & $12(13.04)$ & $61(19.37)$ & Ref. \\
\hline Stages I-II & $174(78.03)$ & $80(86.96)$ & $254(80.63)$ & $1.88(0.95-3.72)$ \\
\hline \multicolumn{5}{|l|}{ Years since diagnosis } \\
\hline$\leq 5$ & $109(48.88)$ & $48(52.17)$ & $157(49.84)$ & Ref. \\
\hline$>5$ & $114(51.12)$ & $44(47.83)$ & $158(50.16)$ & $0.88(0.54-1.43)$ \\
\hline \multicolumn{5}{|l|}{ Radiation } \\
\hline No & $105(47.09)$ & $44(47.83)$ & $149(47.30)$ & Ref. \\
\hline Yes & $118(52.91)$ & $48(52.17)$ & $166(52.70)$ & $0.97(0.60-1.58)$ \\
\hline \multicolumn{5}{|l|}{ Hormonal (Tamoxifen/AIs) } \\
\hline No & $114(51.12)$ & $40(43.48)$ & $154(48.89)$ & Ref. \\
\hline Yes & $109(48.88)$ & $52(56.52)$ & $161(51.11)$ & $1.36(0.83-2.22)$ \\
\hline \multicolumn{5}{|l|}{ Chemotherapy } \\
\hline No & $163(73.09)$ & $59(64.13)$ & $222(70.48)$ & Ref. \\
\hline Yes & $60(26.91)$ & $33(35.87)$ & $93(29.52)$ & $1.52(0.90-2.55)$ \\
\hline \multicolumn{5}{|l|}{ Charlson Comorbidity Index } \\
\hline 0 & $62(27.80)$ & $25(27.17)$ & $87(27.62)$ & Ref. \\
\hline $1-2$ & $89(39.91)$ & $36(39.13)$ & $125(39.68)$ & $1.00(0.55-1.84)$ \\
\hline $3+$ & $72(32.29)$ & $31(33.70)$ & $103(32.70)$ & $1.07(0.57-2.00)$ \\
\hline \multicolumn{5}{|l|}{ Physical activity } \\
\hline Insufficiently active & $46(22.77)$ & $26(29.55)$ & $72(24.83)$ & Ref. \\
\hline Moderately active & $52(25.74)$ & $25(28.41)$ & $77(26.55)$ & $0.85(0.43-1.67)$ \\
\hline Active & $104(51.49)$ & $37(42.05)$ & $141(48.62)$ & $0.63(0.34-1.16)$ \\
\hline Unknown/missing & $21(\mathrm{~N} / \mathrm{A})$ & $4(\mathrm{~N} / \mathrm{A})$ & $25(\mathrm{~N} / \mathrm{A})$ & N/A \\
\hline \multicolumn{5}{|l|}{ RFQ } \\
\hline Mean & 1.67 & 2.08 & 1.79 & \\
\hline Median & 1.36 & 1.82 & 1.46 & $p=0.0003$ \\
\hline Lower, upper quartiles & $1.18-1.82$ & $1.27-2.73$ & $1.18-2.18$ & \\
\hline \multicolumn{5}{|l|}{ PSS } \\
\hline None (0-13) & $82(37.79)$ & $6(6.52)$ & $88(28.48)$ & Ref. \\
\hline Some $(14+)$ & $135(62.21)$ & $86(93.48)$ & $221(71.52)$ & $8.71(3.64-20.82)$ \\
\hline Unknown/missing & 6 (N/A) & 0 (N/A) & 6 (N/A) & N/A \\
\hline \multicolumn{5}{|l|}{ Vasomotor symptoms } \\
\hline No & $96(44.44)$ & $40(44.44)$ & $136(44.44)$ & Ref. \\
\hline Yes & $120(55.56)$ & $50(55.56)$ & $170(55.56)$ & $1.00(0.61-1.64)$ \\
\hline Unknown/missing & 7 (N/A) & 2 (N/A) & 9 (N/A) & N/A \\
\hline \multicolumn{5}{|l|}{ Lifetime depression history } \\
\hline No & $156(69.96)$ & $55(59.78)$ & $211(66.98)$ & Ref. \\
\hline Yes & $67(30.04)$ & $37(40.22)$ & $104(33.02)$ & $1.57(0.94-2.60)$ \\
\hline \multicolumn{5}{|l|}{ Lifetime insomnia history } \\
\hline No & $175(78.48)$ & $44(47.83)$ & $219(69.52)$ & Ref. \\
\hline Yes & $48(21.52)$ & $48(52.17)$ & $96(30.48)$ & $3.98(2.37-6.68)$ \\
\hline \multicolumn{5}{|l|}{ Antidepressants (use post-BC dx) } \\
\hline No & $194(87.00)$ & $67(72.83)$ & $261(82.86)$ & Ref. \\
\hline Yes & $29(13.00)$ & $25(27.17)$ & $54(17.14)$ & $2.50(1.37-4.56)$ \\
\hline \multicolumn{5}{|l|}{ Sleep medication (use post-BC $\mathrm{dx}$ ) } \\
\hline No & $190(85.20)$ & $73(79.35)$ & $263(83.49)$ & Ref. \\
\hline Yes & $33(14.80)$ & $19(20.65)$ & $52(16.51)$ & $1.50(0.80-2.80)$ \\
\hline
\end{tabular}

$p$-Values and percents are based on known values.

IDS, Inventory of Depressive Symptoms. 
Table 3. Demographics, Clinical, and Tumor Characteristics by Baseline Fatigue Symptom Inventory

\begin{tabular}{|c|c|c|c|c|}
\hline Total & $\begin{array}{c}\text { None } \\
(\text { FSI <3 or unknown }) \\
\mathrm{N}=194, \mathrm{~N}(\%)\end{array}$ & $\begin{array}{l}\text { Some }(F S I \geq 3), \\
\mathrm{N}=121, \mathrm{~N}(\%)\end{array}$ & $\begin{array}{l}\text { Total, 315, } \\
\quad \mathrm{N}(\%)\end{array}$ & $\begin{array}{c}\text { Overall OR } \\
(95 \% C I)\end{array}$ \\
\hline \multicolumn{5}{|l|}{ Age at interview (years) } \\
\hline$\leq 65$ & $32(16.49)$ & $27(22.31)$ & $59(18.73)$ & Ref. \\
\hline$>65$ & $162(83.51)$ & $94(77.69)$ & $256(81.27)$ & $0.69(0.39-1.23)$ \\
\hline \multicolumn{5}{|l|}{ Race/ethnicity } \\
\hline White (Hispanic and non-Hispanic) & $135(69.58)$ & $86(71.07)$ & $221(70.16)$ & Ref. \\
\hline African American & $40(20.62)$ & $26(21.49)$ & $66(20.95)$ & $1.02(0.55-1.85)$ \\
\hline Asian & $19(9.79)$ & $9(7.44)$ & $28(8.89)$ & $0.74(0.28-1.82)$ \\
\hline \multicolumn{5}{|l|}{ Stage at $\mathrm{BC} d x$} \\
\hline Stage 0 (DCIS) & $44(22.68)$ & $17(14.05)$ & $61(19.37)$ & Ref. \\
\hline Stages I-II & $150(77.32)$ & $104(85.95)$ & $254(80.63)$ & $1.79(0.97-3.12)$ \\
\hline \multicolumn{5}{|l|}{ Years since diagnosis } \\
\hline$\leq 5$ & $88(45.36)$ & $69(57.02)$ & $157(49.84)$ & Ref. \\
\hline$>5$ & $106(54.64)$ & $52(42.98)$ & $158(50.16)$ & $0.63(0.40-0.99)$ \\
\hline \multicolumn{5}{|l|}{ Radiation } \\
\hline No & $92(47.42)$ & $57(47.11)$ & $149(47.30)$ & Ref. \\
\hline Yes & $102(52.58)$ & $64(52.89)$ & $166(52.70)$ & $1.01(0.64-1.60)$ \\
\hline \multicolumn{5}{|l|}{ Hormonal (Tamoxifen/AIs) } \\
\hline No & $102(52.58)$ & $52(42.98)$ & $154(48.89)$ & Ref. \\
\hline Yes & $92(47.42)$ & $69(57.02)$ & $161(51.11)$ & $1.47(0.93-2.32)$ \\
\hline \multicolumn{5}{|l|}{ Chemotherapy } \\
\hline No & $144(74.23)$ & $78(64.46)$ & $222(70.48)$ & Ref. \\
\hline Yes & $50(25.77)$ & $43(35.54)$ & $93(29.52)$ & $1.59(0.97-2.60)$ \\
\hline \multicolumn{5}{|l|}{ Charlson Comorbidity Index } \\
\hline 0 & $61(31.44)$ & $26(21.49)$ & $87(27.62)$ & Ref. \\
\hline $1-2$ & $72(37.11)$ & $53(43.80)$ & $125(39.68)$ & $0.58(0.32-1.03)$ \\
\hline $3+$ & $61(31.44)$ & $42(34.71)$ & $103(32.70)$ & $0.62(0.34-1.13)$ \\
\hline \multicolumn{5}{|l|}{ Physical activity } \\
\hline Insufficiently active & $30(17.34)$ & $42(35.90)$ & $72(24.83)$ & Ref. \\
\hline Moderately active & $51(29.48)$ & $26(22.22)$ & $77(26.55)$ & $2.75(1.41-5.43)$ \\
\hline Active & $92(53.18)$ & $49(41.88)$ & $141(48.62)$ & $2.63(1.47-4.71)$ \\
\hline Unknown/missing & 21 (NA) & 4 (NA) & 25 (NA) & N/A \\
\hline \multicolumn{5}{|l|}{ RFQ } \\
\hline Mean & 1.68 & 1.95 & 1.79 & \\
\hline Median & 1.36 & 1.55 & 1.45 & $p=0.0102$ \\
\hline Lower, upper quartiles & $1.18-1.91$ & $1.18-2.50$ & $1.18-2.18$ & \\
\hline \multicolumn{5}{|l|}{ PSS } \\
\hline None (0-13) & $70(36.84)$ & $18(15.13)$ & $88(28.48)$ & Ref. \\
\hline Some $(14+)$ & $120(63.16)$ & $101(84.87)$ & $221(71.52)$ & $3.27(1.85-5.86)$ \\
\hline Unknown/missing & 4 (NA) & 2 (NA) & 6 (NA) & N/A \\
\hline \multicolumn{5}{|l|}{ Vasomotor symptoms } \\
\hline No & $85(43.81)$ & $51(42.15)$ & $136(44.44)$ & Ref. \\
\hline Yes & $103(53.09)$ & $67(55.37)$ & $170(55.56)$ & $1.08(0.68-1.72)$ \\
\hline Unknown/missing & 6 (NA) & 3 (NA) & 9 (NA) & N/A \\
\hline \multicolumn{5}{|l|}{ Lifetime depression history } \\
\hline No & $138(71.13)$ & $73(60.33)$ & $211(66.98)$ & Ref. \\
\hline Yes & $56(28.87)$ & $48(39.67)$ & $104(33.02)$ & $1.62(1.00-2.62)$ \\
\hline \multicolumn{5}{|l|}{ Lifetime insomnia history } \\
\hline No & $152(78.35)$ & $67(55.37)$ & $219(69.52)$ & Ref. \\
\hline Yes & $42(21.65)$ & $54(44.63)$ & $96(30.48)$ & $2.92(1.78-4.79)$ \\
\hline \multicolumn{5}{|l|}{ Antidepressants (use post-BC dx) } \\
\hline No & $164(85.54)$ & $87(71.90)$ & $251(79.68)$ & Ref. \\
\hline Yes & $20(10.31)$ & $34(28.10)$ & $54(17.14)$ & $3.21(1.74-5.90)$ \\
\hline \multicolumn{5}{|l|}{ Sleep medication (use post-BC $\mathrm{dx}$ ) } \\
\hline No & $166(85.57)$ & $97(80.17)$ & $263(83.49)$ & Ref. \\
\hline Yes & $28(14.43)$ & $24(19.83)$ & $52(16.51)$ & $1.47(0.81-2.67)$ \\
\hline
\end{tabular}

$p$-Values and percents are based on known values. FSI, Fatigue Symptom Inventory. 
Table 4. Multivariable Adjusted Odds Ratios for the Association Between Inventory of Depressive Symptomology and Insomnia Severity Index and Other Correlates

\begin{tabular}{|c|c|c|c|c|c|}
\hline Correlates & Category & Crude OR $(95 \%$ CI $)$ & $\mathrm{p}$ & Adjusted $O R^{\mathrm{a}}(95 \%$ CI $)$ & $\mathrm{p}$ \\
\hline ISI & None (ISI $<8$ or unknown) & Ref. & & Ref. & \\
\hline \multirow[t]{2}{*}{ Lifetime depression $\mathrm{Hx}$} & $\begin{array}{l}\text { Some }(I S I \geq 8) \\
\text { No }\end{array}$ & $\begin{array}{c}7.63 \text { (4.44-13.10) } \\
\text { Ref. }\end{array}$ & $<0.0001$ & $\begin{array}{c}5.98 \text { (3.04-11.76) } \\
\text { Ref. }\end{array}$ & $<0.0001$ \\
\hline & Yes & $1.57(0.95-1.60)$ & 0.08 & $0.78(0.39-1.57)$ & 0.49 \\
\hline Lifetime insomnia $\mathrm{Hx}$ & $\begin{array}{l}\text { No } \\
\text { Yes }\end{array}$ & $\begin{array}{c}\text { Ref. } \\
3.98 \text { (2.37-6.68) }\end{array}$ & $<0.0001$ & $\begin{array}{l}\text { Ref. } \\
2.01(1.03-3.94)\end{array}$ & 0.04 \\
\hline \multirow[t]{2}{*}{ Age at interview (years) } & $\leq 65$ & Ref. & & Ref. & \\
\hline & $>65$ & $0.93(0.50-1.72)$ & 0.81 & $1.34(0.59-3.03)$ & 0.49 \\
\hline Years since $\mathrm{BC}$ diagnosis & $\begin{array}{l}\leq 5 \\
>5\end{array}$ & $\begin{array}{c}\text { Ref. } \\
0.88(0.54-1.43)\end{array}$ & 0.59 & $\begin{array}{c}\text { Ref. } \\
0.67(0.35-1.26)\end{array}$ & 0.21 \\
\hline \multirow[t]{3}{*}{ Race/ethnicity } & $\begin{array}{l}\text { White, Hispanic } \\
\text { and non-Hispanic }\end{array}$ & Ref. & & Ref. & \\
\hline & African American/black & $1.17(0.65-2.12)$ & 0.65 & $2.04(0.96-4.35)$ & 0.35 \\
\hline & Asian & $1.00(0.42-2.40)$ & 0.87 & $1.84(0.55-6.18)$ & 0.67 \\
\hline \multirow{2}{*}{$\begin{array}{l}\text { Antidepressants } \\
\text { (ever use post-BC dx) }\end{array}$} & No & Ref. & & Ref. & \\
\hline & Yes & $2.50(1.37-4.56)$ & 0.0029 & $3.11(1.38-7.05)$ & 0.01 \\
\hline \multirow{2}{*}{$\begin{array}{l}\text { Sleep medication use } \\
\text { (ever use post-BC } \mathrm{dx})\end{array}$} & No & Ref. & & Ref. & \\
\hline & Yes & $1.50(0.80-2.80)$ & 0.21 & $0.52(0.23-1.17)$ & 0.11 \\
\hline \multirow{3}{*}{$\begin{array}{l}\text { Initial stage at BC } \\
\text { diagnosis } \\
\text { PSS }\end{array}$} & $\begin{array}{l}\text { Stage } 0 \text { (DCIS) } \\
\text { Stages I-II }\end{array}$ & $\begin{array}{l}\text { Ref. } \\
188(095-372)\end{array}$ & 007 & $\begin{array}{l}\text { Ref. } \\
175(076-404)\end{array}$ & 0.19 \\
\hline & None & $\begin{array}{l}1.88 \text { (0.9J-3.I2) } \\
\text { Ref. }\end{array}$ & & $\begin{array}{l}\text { 1./5 (0.10-4. } \\
\text { Ref. }\end{array}$ & 0.13 \\
\hline & Some & $9.34(3.91-22.30)$ & $<0.0001$ & $6.37(2.49-16.33)$ & $<0.000$ \\
\hline RFQ & Continuous & $1.73(1.30-2.30)$ & 0.0002 & $1.79(1.26-2.56)$ & 0.00 \\
\hline
\end{tabular}

${ }^{a}$ Adjusted for all variables listed in table.

diagnosis, race/ethnicity, ever antidepressant and sleep medication use, lifetime depression history, and lifetime insomnia history (Table 4), and current perceived stress. Of these covariates, lifetime insomnia history (adjusted $\mathrm{OR}=2.01,95 \% \mathrm{CI}:$ 1.03-3.94), ever antidepressant use (adjusted OR $=3.11,95 \%$ CI: $1.38-7.05$ ), and greater current perceived stress (adjusted OR $=6.37,95 \%$ CI: 2.49-16.33) were independently associated with current depressive symptoms. Early childhood stress was also correlated with current depressive symptoms $(p=0.0013)$. Further, the odds of current depressive symptoms increased by $80 \%$ with a one unit increase in the RFQ score (adjusted OR $=1.79,95 \% \mathrm{CI}$ : 1.25-2.56). These multivariable results suggest despite ever use of antidepressants after breast cancer diagnosis, women still reported high occurrence of current depressive symptoms, furthermore, lifetime history of insomnia was associated with their current depression status. However, a lifetime depression history was not correlated with current depressive symptoms.

\section{Insomnia symptoms (ISI >8) and fatigue occurrence (FSI >3)}

Nearly $38 \%(121 / 315)$ of the survivors reported current fatigue (FSI $\geq 3$ score) (Table 3 ). In bivariate analyses, the strongest correlates of current fatigue included perception of stress (OR $=3.27,95 \% \mathrm{CI}: 1.65-5.86)$; ever use of antidepressants $(\mathrm{OR}=3.21,95 \% \mathrm{CI}: 1.74-5.90)$; lifetime depression history $(\mathrm{OR}=1.62,95 \% \mathrm{CI}$ : $1.00-2.62)$; and lifetime insomnia history ( $\mathrm{OR}=2.92,95 \% \mathrm{CI}: 1.78-4.79)$. Interestingly, ever use of sleep aids was associated with $47 \%$ higher odds of current fatigue, but the association was not statistically significant $(\mathrm{OR}=1.47,95 \% \mathrm{CI}$ : $0.81-2.67)$.
Odds of current fatigue was over sixfold greater in survivors who reported current insomnia symptoms (unadjusted $\mathrm{OR}=6.03$, 95\% CI: 3.66-10.71); this association persisted after multivariable adjustment (adjusted $\mathrm{OR}=5.02,95 \% \mathrm{CI}$ : 2.67-9.44) (Table 5). In the multivariable model, the odds of current fatigue were fourfold greater in women who ever used antidepressants (adjusted OR $=4.52$, 95\% CI: 2.12-9.64) versus those who never used these medications. Interestingly, long-term survivors of 5 years or more (adjusted $\mathrm{OR}=0.44$, 95\% CI: $0.25-0.79$ ) were half likely to report current fatigue than more recently diagnosed survivors. Other variables such as lifetime insomnia history, lifetime depression history, age at interview, race/ethnicity, ever sleep medication use, and breast cancer stage were not statistically associated with current fatigue.

\section{Discussion}

Overall, in breast cancer survivors who had completed their primary cancer treatment over 2 years ago, the prevalence of current depressive symptoms was nearly $30 \%$ and the prevalence of current fatigue was $38 \%$. These results are consistent with studies that determined the prevalence of depressive symptoms ranged from $20 \%$ to $30 \%$ in community-dwelling breast cancer survivors. ${ }^{5,13,48}$ Of note, our results suggest that breast cancer survivors with current insomnia symptoms have a sixfold greater odds of reporting current depressive symptoms and a fivefold increased odds of experiencing fatigue. In addition, having ever used antidepressants or sleep medications was not associated with lower odds of current depressive symptoms or lower current fatigue. This result is consistent with a meta-analysis that demonstrated that the effectiveness of antidepressants versus 
Table 5. Multivariable Adjusted Odds Ratios for the Association Between Fatigue Symptom Inventory, Insomnia Severity Index, AND Other Correlates

\begin{tabular}{|c|c|c|c|c|c|}
\hline Correlates & Category & Crude OR $(95 \%$ CI $)$ & $\mathrm{p}$ & Adjusted $O R^{\mathrm{a}}(95 \%$ CI $)$ & $\mathrm{p}$ \\
\hline ISI & None (ISI $<8$ or unknown) & Ref. & & Ref. & \\
\hline \multirow{2}{*}{ Lifetime depression $\mathrm{Hx}$} & $\begin{array}{l}\text { Some (ISI } \geq 8) \\
\text { No }\end{array}$ & $\begin{array}{c}6.03(3.61-10.07) \\
\text { Ref. }\end{array}$ & $<0.0001$ & $5.02 \underset{\text { Ref }}{(2.67-9.44)}$ & $<0.0001$ \\
\hline & Yes & $1.62(1.00-2.62)$ & 0.05 & $0.85(0.46-1.58)$ & 0.60 \\
\hline \multirow[t]{2}{*}{ Lifetime insomnia $\mathrm{Hx}$} & No & Ref. & & Ref. & \\
\hline & Yes & $2.92(1.78-4.79)$ & $<0.0001$ & $1.41(0.74-2.69)$ & 0.29 \\
\hline \multirow[t]{2}{*}{ Age at interview } & $<65$ & Ref. & & Ref. & \\
\hline & $\begin{array}{l}>65 \\
\leq 5\end{array}$ & $\begin{array}{c}0.69 \text { (0.39-1.22) } \\
\text { Ref. }\end{array}$ & 0.20 & $\begin{array}{c}0.85 \text { (0.41-1.74) } \\
\text { Ref. }\end{array}$ & 0.66 \\
\hline Years since $\mathrm{BC}$ diagnosis & $>5$ & $0.63(0.40-0.99)$ & 0.05 & $0.45(0.25-0.79)$ & 0.01 \\
\hline \multirow[t]{3}{*}{ Race/ethnicity } & $\begin{array}{l}\text { White, Hispanic and } \\
\text { non-Hispanic }\end{array}$ & Ref. & & Ref. & \\
\hline & African American/black & $1.02(0.58-1.79)$ & 0.61 & $1.42(0.73-2.77)$ & 0.36 \\
\hline & Asian & $0.75(0.32-1.72)$ & 0.48 & $0.99(0.35-2.82)$ & 0.73 \\
\hline \multirow{2}{*}{$\begin{array}{l}\text { Antidepressants } \\
\text { (ever use post-BC dx) }\end{array}$} & No & Ref. & & Ref. & \\
\hline & Yes & $3.40(1.85-6.25)$ & $<0.0001$ & $4.52(2.12-9.64)$ & $<0.0001$ \\
\hline \multirow{2}{*}{$\begin{array}{l}\text { Sleep medication use } \\
\text { (ever use post-BC } \mathrm{dx} \text { ) }\end{array}$} & No & Ref. & & Ref. & \\
\hline & $\begin{array}{l}\text { Yes } \\
\text { Stage } 0 \text { (DCIS) }\end{array}$ & $\begin{array}{c}1.47(0.81-2.67) \\
\text { Ref. }\end{array}$ & 0.21 & $0.66(0.31-1.38)$ & 0.27 \\
\hline $\begin{array}{l}\text { Initial stage at } \mathrm{BC} \\
\text { diagnosis }\end{array}$ & $\begin{array}{l}\text { Stage } 0 \text { (DCIS) } \\
\text { Stages I-II }\end{array}$ & 1.79 (0.97-3.31) & 0.06 & $\begin{array}{c}\text { Ref. } \\
1.56(0.77-3.18)\end{array}$ & 0.22 \\
\hline \multirow[t]{2}{*}{ PSS } & None & Ref. & & Ref. & \\
\hline & Some & $3.11(1.78-5.45)$ & $<0.0001$ & $1.79(0.94-3.40)$ & 0.08 \\
\hline RFQ & Continuous & $1.45(1.10-1.90)$ & 0.008 & $1.46(1.05-2.03)$ & 0.02 \\
\hline
\end{tabular}

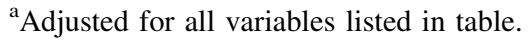

placebo is minimal or nonexistent in adults with mild or moderate depressive symptoms (as in majority of participants in the present study), although such medications may alleviate symptoms in those with severe depression. ${ }^{49}$ Further, having a history of lifetime insomnia was associated with current depressive symptoms, although history of lifetime depression was not correlated with such symptoms. This finding is consistent with a meta-analysis of prospective studies that determined that insomnia is strongly correlated with an increased depression in those who did not have comorbid depression. ${ }^{50}$ It is possible that lifetime depression history was not strongly correlated with current depression symptoms because the prior episodes occurred long before cancer diagnosis.

Regarding clinical implications, our study suggests that assessing insomnia should be incorporated into cancer survivorship care plans, with possible recommendation of cognitive behavioral therapy to reduce insomnia; this may potentially mitigate depressive and fatigue symptoms during survivorship. ${ }^{42,51,52}$ Antidepressants are prescribed to breast cancer survivors to cope with hot flashes, depressive symptoms, or sleep problems. However, we found that depressive symptoms and fatigue persisted among women who ever used antidepressants any time after breast cancer diagnosis. Although it is not surprising as more patients with depression are more likely to be prescribed antidepressants, the threefold magnitude of the association suggests that behavioral therapy may help alleviate depressive symptoms, rather than solely using pharmaceuticals. Past use of antidepressants and sleep aids may be correlated with current and future use. It is possible that although some women had long-term intermittent exposure to these medications throughout their survivorship period (including a fraction who might have recently used these medications), women still reported high prevalence of current insomnia symptoms (33\%), depressive symptoms (29\%), and feeling fatigued (38\%). Thus, future studies should focus on alleviating insomnia symptoms via behavioral interventions to lower the risk of new depressive episodes; this needs to be confirmed in larger studies with longitudinal data and considering more stringent cut points for the ISI questionnaire (e.g., $>10) .{ }^{41}$ Further, insomnia symptoms, depression, and fatigue may exist as a symptom cluster in breast cancer survivors, and therefore, a combination of behavioral and pharmacologic treatments might improve overall quality of life..$^{53,54}$

Similar to our study, Reich et al. also found that risk factors for depression after breast cancer diagnosis are more related to the patient rather than to the disease or its treatment. ${ }^{55} \mathrm{In}$ their review, breast cancer stage was not significantly associated with distress. Because depression affects other quality of life domains such as fatigue and attitudes of helplessness/hopelessness, managing depressive symptoms in breast cancer survivors would enhance overall psychosocial health.

Our study has a number of strengths. Our cohort was diverse and 30\% of the group was African American or Asian/Pacific Islanders, and the response rate among the eligible phone screened women was high (84.9\%). Regarding racial/ethnic distribution, our cohort included nearly three times more African American women (21\%) in comparison to California, which reported 7\% African Americans diagnosed with breast cancer in $2017 .{ }^{56}$ This enhances the study's generalizability. Regarding antidepressant and sleep medication use, we asked if women used these drugs since their breast cancer diagnosis, and because the median years since cancer diagnosis was 6 years, our results suggest that these medications were initiated years before the baseline 
interview; this helps address the temporality of the associations we found with current depressive symptoms and current fatigue. Further, all women were recruited from one large health plan ensuring that participants had similar health care access, and therefore, bias resulting from variable medical coverage is low. We excluded women with a prevalent (unremitted) major depression disorder, an important confounder. Also, the psychosocial outcomes were collected during proctored interviews and we used validated instruments. We were able to adjust for multiple covariates rarely accounted for in prior studies; cancer treatments, tumor characteristics, comorbidity, and sociodemographic information were captured from patients' EMR.

Some limitations must be considered. Although selection bias is possible with women with less severe depression participating in the study, the clinical characteristics of the breast cancer survivors who participated in the study was similar to the larger KPSC population in terms of race/ ethnicity, stage of diagnosis, receipt of adjuvant treatments, and comorbidity status. For example, nearly $72 \%$ had a Charlson comorbidity index of two or more. Next steps include confirming if poor sleep quality is associated with future depressive symptoms based on our longitudinal 32month prospective study. Given that women with unremitted depression were excluded at screening, this might have limited the generalizability of our results to the general community of breast cancer survivors. In addition, given the cross-sectional design, depressive symptoms might have fueled insomnia symptoms, or insomnia symptoms might have led to depression symptoms.

Further, we were not able to examine anxiety occurrence in this population because very few women $(N=5)$ reported such symptoms. Anxiety may be prodromal to depressive symptoms, thus, we may have underascertained depression. Another limitation is that we could not distinguish women who were currently taking the study medications from past users. Thus, it is possible that we could have found statistically significant lower odds of current depressive symptoms and current fatigue in women who recently used such medications. However, based the strength of the associations we observed between ever antidepressant use and current depressive symptoms (adjusted OR 3.11, 95\% CI: 1.38-7.05) and with current fatigue (adjusted OR 4.52, 95\% CI: $2.12-$ 9.64), we expect that these ORs would have been even stronger in the subset of women who were currently taking antidepressants. Furthermore, we could not assess psychotherapy as a covariate in our analysis, but checking against the EMR, it appeared that participants had not received psychotherapy.

\section{Conclusion}

In this cohort of breast cancer survivors, our results suggest that insomnia symptoms are strongly correlated with current depressive symptoms and fatigue, even after accounting for lifetime depression history. Moreover, lifetime insomnia was more strongly correlated with these conditions. Ever use of antidepressants or sleep medications was not correlated with lower occurrence of depressive symptoms and fatigue in those with insomnia symptoms. The clinical implications of this study suggest that cancer survivorship care plans should incorporate screening for insomnia symptoms and surveil- lance of psychosocial needs, and that behavioral therapy might help improve sleep problems that may in turn reduce depression and fatigue symptoms ${ }^{57-63}$; however, this needs to be confirmed with longitudinal data.

\section{Data Availability}

The deidentified datasets generated from this analysis are available from the corresponding author's pending data use agreements and IRB approvals from KPSC and UCLA. In addition, funding may be required for data transfer.

\section{Compliance with Ethical Standards}

The study was reviewed and approved by the KPSC IRB of Kaiser Permanente (KPSC) and the UCLA. The study obtained written informed consent from all participants. All study procedures followed the standards of the KPSC and UCLA IRBs and with the Declaration of Helsinki 1975 and its later amendments or comparable ethical standards.

\section{Acknowledgments}

The authors thank the patients of Kaiser Permanente for helping improve care through their participation and thank the dedicated research staff at KPSC and UCLA.

\section{Author Disclosure Statement}

The authors declare no conflict of interest.

\section{Funding Information}

This project was supported by National Institutes of Health/National Cancer Institute R01CA160245 and R01CA207130. The content is solely the responsibility of the authors and does not necessarily represent the official views of the National Institutes of Health.

\section{References}

1. Ford DE, Kamerow DB. Epidemiologic study of sleep disturbances and psychiatric disorders. An opportunity for prevention? JAMA 1989;262:1479-1484.

2. Elenkov IJ, Wilder RL, Chrousos GP, Vizi ES. The sympathetic nerve-An integrative interface between two supersystems: The brain and the immune system. Pharmacol Rev 2000;52:595-638.

3. NCI Office of Cancer Research. Statistics. Available at: https:// cancercontrol.cancer.gov/ocs/statistics. Accessed June 1, 2020.

4. Miller AH, Ancoli-Israel S, Bower JE, et al. Neuroendocrineimmune mechanisms of behavioral comorbidities in patients with cancer. J Clin Oncol 2008;26:971-982.

5. Massie MJ. Prevalence of depression in patients with cancer. J Natl Cancer Inst Monogr 2004;32:57-71.

6. Fann JR, Thomas-Rich AM, Katon WJ, et al. Major depression after breast cancer: A review of epidemiology and treatment. Gen Hosp Psychiatry 2008;30:112-126.

7. Raison CL, Miller AH. Depression in cancer: New developments regarding diagnosis and treatment. Biol Psychiatry 2003;54:283-294.

8. Evans DL, Charney DS, Lewis L, et al. Mood disorders in the medically ill: Scientific review and recommendations. Biol Psychiatry 2005;58:175-189. 
9. Spiegel D, Giese-Davis J. Depression and cancer: Mechanisms and disease progression. Biol Psychiatry 2003;54: 269-282.

10. Polsky D, Doshi JA, Marcus S, et al. Long-term risk for depressive symptoms after a medical diagnosis. Arch Intern Med 2005;165:1260-1266.

11. Institute of Medicine. Cancer care for the whole patient: Meeting psychosocial health needs. Washington, DC: Institute of Medicine of the National Academies, 2007.

12. Institute of Medicine. Meeting the psychosocial needs of women with breast cancer. Washington, DC: Institute of Medicine of the National Academies, 2004.

13. Cho HJ, Lavretsky H, Olmstead R, Levin MJ, Oxman MN, Irwin MR. Sleep disturbance and depression recurrence in community-dwelling older adults: A prospective study. Am J Psychiatry 2008;165:1543-1550.

14. Cole MG, Dendukuri N. Risk factors for depression among elderly community subjects: A systematic review and metaanalysis. Am J Psychiatry 2003;160:1147-1156.

15. Hill J, Holcombe C, Clark L, et al. Predictors of onset of depression and anxiety in the year after diagnosis of breast cancer. Psychol Med 2011;41:1429-1436.

16. Savard J, Morin CM. Insomnia in the context of cancer: A review of a neglected problem. J Clin Oncol 2001;19:895908.

17. Fiorentino L, Ancoli-Israel S. Insomnia and its treatment in women with breast cancer. Sleep Med Rev 2006;10:419-429.

18. Savard J, Simard S, Blanchet J, Ivers H, Morin CM. Prevalence, clinical characteristics, and risk factors for insomnia in the context of breast cancer. Sleep 2001;24:583-590.

19. Palesh OG, Roscoe JA, Mustian KM, et al. Prevalence, demographics, and psychological associations of sleep disruption in patients with cancer: University of Rochester Cancer Center-Community Clinical Oncology Program. J Clin Oncol 2010;28:292-298.

20. Amiel CR, Fisher HM, Antoni MH. Concerns about breast cancer, pain, and fatigue in non-metastatic breast cancer patients undergoing primary treatment. Healthcare (Basel) 2016;4:62.

21. Walker J, Holm Hansen C, Martin P, et al. Prevalence of depression in adults with cancer: A systematic review. Ann Oncol 2013;24:895-900.

22. Pinquart M, Duberstein PR. Depression and cancer mortality: A meta-analysis. Psychol Med 2010;40:1797-1810.

23. Irwin MR. Depression and insomnia in cancer: Prevalence, risk factors, and effects on cancer outcomes. Curr Psychiatry Rep 2013;15:404.

24. World Health Organization. Cancer. Available at: www .who.int/mediacentre/factsheets/fs297/es/2018 Accessed January 15, 2020.

25. Calderon C, Carmona-Bayonas A, Hernández R, et al. Effects of pessimism, depression, fatigue, and pain on functional health-related quality of life in patients with resected non-advanced breast cancer. Breast 2019;44:108-112.

26. Coughlin SS, Yoo W, Whitehead MS, Smith SA. Advancing breast cancer survivorship among African-American women. Breast Cancer Res Treat 2015;153:253-261.

27. Johns SA, Stutz PV, Talib TL, et al. Acceptance and commitment therapy for breast cancer survivors with fear of cancer recurrence: A 3-arm pilot randomized controlled trial. Cancer 2020;126:211-218.

28. Bedillion MF, Ansell EB, Thomas GA. Cancer treatment effects on cognition and depression: The moderating role of physical activity. Breast 2019;44:73-80.
29. Baker F, Denniston M, Smith T, West MM. Adult cancer survivors: How are they faring? Cancer 2005;104(11 Suppl): 2565-2576.

30. Jim HS, Phillips KM, Chait S, et al. Meta-analysis of cognitive functioning in breast cancer survivors previously treated with standard-dose chemotherapy. J Clin Oncol 2012;30:3578-3587.

31. Budhrani PH, Lengacher CA, Kip KE, et al. Minority breast cancer survivors: The association between race/ethnicity, objective sleep disturbances, and physical and psychological symptoms. Nurs Res Pract 2014;2014:858403.

32. Ferreira AR, Di Meglio A, Pistilli B, et al. Differential impact of endocrine therapy and chemotherapy on quality of life of breast cancer survivors: A prospective patientreported outcomes analysis. Ann Oncol 2019;30:17841795.

33. $\mathrm{Ng}$ HS, Vitry A, Koczwara B, Roder D, McBride ML. Patterns of comorbidities in women with breast cancer: A Canadian population-based study. Cancer Causes Control 2019;30:931-941.

34. McFarland DC, Andreotti C, Harris K, Mandeli J, Tiersten A, Holland J. Early childhood adversity and its associations with anxiety, depression, and distress $\mathrm{n}$ women with breast cancer. Psychosomatics 2016;57:174-184.

35. Seib C, Porter-Steele J, Ng SK, et al. Life stress and symptoms of anxiety and depression in women after cancer: The mediating effect of stress appraisal and coping. Psychooncology 2018;27:1787-1794.

36. Beatty L, Lee C, Wade TD. A prospective examination of perceived stress as a mediator of the relationship between life-events and QOL following breast cancer. Br J Health Psychol 2009;14:789-804.

37. First MB, Williams JBW, Karg RS, Spitzer RL. Structured clinical interview for DSM-5-Research version (SCID-5 for DSM-5, research version; SCID-5-RV). Arlington, VA: American Psychiatric Association, 2015.

38. Rush AJ, Gullion CM, Basco MR, Jarrett RB, Trivedi MH. The Inventory of Depressive Symptomatology (IDS): Psychometric properties. Psychol Med 1996;26:477-486.

39. Hann DM, Jacobsen PB, Azzarello LM, et al. Measurement of fatigue in cancer patients: Development and validation of the Fatigue Symptom Inventory. Qual Life Res 1998;7: 301-310.

40. Cohen S, Kamarck T, Mermelstein R. A global measure of perceived stress. J Health Soc Behav 1983;24:385-396.

41. Morin CM, Belleville G, Bélanger L, Ivers H. The Insomnia Severity Index: Psychometric indicators to detect insomnia cases and evaluate treatment response. Sleep 2011;34:601-608.

42. Schramm E, Hohagen F, Grasshoff U, et al. Test-retest reliability and validity of the Structured Interview for Sleep Disorders according to DSM-III-R. Am J Psychiatry 1993; 150:867-872.

43. Taylor SE, Lerner JS, Sage RM, Lehman BJ, Seeman TE. Early environment, emotions, responses to stress, and health. J Pers 2004;72:1365-1393.

44. Felitti VJ, Anda R, Nordenberg D, et al. Relationship of childhood abuse and household dysfunction to many of the leading causes of death in adults. Am J Prev Med 1998;14: 245-258.

45. Godin G. The Godin-Shephard Leisure-Time Physical Activity Questionnaire. Health Fit J Can 2011;4:18-22.

46. Carpenter JS. The Hot Flash Related Daily Interference Scale: A tool for assessing the impact of hot flashes on 
quality of life following breast cancer. J Pain Symptom Manage 2001;22:979-989.

47. Cleves AM, Sanchez N, Draheim N. Evaluation of two competing methods for calculating Charlson's comorbidity index when analyzing short-term mortality using administrative data. J Clin Epidemiol 1997:50;903-908.

48. Bower JE. Behavioral symptoms in breast cancer patients and survivors: Fatigue, insomnia, depression, and cognitive disturbance. J Clin Oncol 2008;26:768-777.

49. Fournier JC, DeRubeis RJ, Hollon SD, et al. Antidepressant drug effects and depression severity: A patient-level metaanalysis. JAMA 2010;303:47-53.

50. Li L, Wu C, Gan Y, et al. Insomnia and the risk of depression: A meta-analysis of prospective cohort studies. BMC Psychiatry 2016;16:375.

51. Savard J, Simard S, Ivers H, Morin CM. Randomized study on the efficacy of cognitive-behavioral therapy for insomnia secondary to breast cancer, part I: Sleep and psychological effects. J Clin Oncol 2005;23:6083-6096.

52. Fleming L, Randell K, Harvey CJ, Espie CA. Does cognitive behaviour therapy for insomnia reduce clinical levels of fatigue, anxiety and depression in cancer patients? Psychooncology 2014;23:679-684.

53. Fiorentino L, Rissling M, Liu L, Ancoli-Israel S. The symptom cluster of sleep, fatigue and depressive symptoms in breast cancer patients: Severity of the problem and treatment options. Drug Discov Today Dis Models 2011;8: 167-173.

54. Donovan KA, Jacobsen PB. Fatigue, depression, and insomnia: Evidence for a symptom cluster in cancer. Semin Oncol Nurs 2007;23:127-135.

55. Reich M, Lesur A, Perdrizet-Chevallier C. Depression, quality of life and breast cancer: A review of the literature. Breast Cancer Res Treat 2008;110:9-17.

56. American Cancer Society, California Department of Public Health, California Cancer Registry. California cancer facts \& figures 2017. Alameda, CA: American Cancer Society, Inc., California Division, 2017.
57. Kline RM, Arora NK, Bradley CJ, et al. Long-term survivorship care after cancer treatment-Summary of a 2017 National Cancer Policy Forum Workshop. J Natl Cancer Inst 2018;110:1300-1310.

58. Morin CM, Bootzin RR, Buysse DJ, Edinger JD, Espie CA, Lichstein KL. Psychological and behavioral treatment of insomnia: Update of the recent evidence (1998-2004). Sleep 2006;29:1398-1414.

59. Reyes-Gibby CC, Anderson KO, Morrow PK, Shete S, Hassan S. Depressive symptoms and health-related quality of life in breast cancer survivors. J Womens Health (Larchmt) 2012;21:311-318.

60. Janz NK, Mujahid M, Chung LK, et al. Symptom experience and quality of life of women following breast cancer treatment. J Womens Health (Larchmt) 2007;16:13481361.

61. Kang DH, Park NJ, McArdle T. Cancer-specific stress and mood disturbance: Implications for symptom perception, quality of life, and immune response in women shortly after diagnosis of breast cancer. ISRN Nurs 2012;2012:608039.

62. Eyles C, Leydon GM, Hoffman CJ, et al. Mindfulness for the self-management of fatigue, anxiety, and depression in women with metastatic breast cancer: A mixed methods feasibility study. Integr Cancer Ther 2015;14:42-56.

63. Shin JA, El-Jawahri A, Parkes A, Schleicher SM, Knight HP, Temel JS. Quality of life, mood, and prognostic understanding in patients with metastatic breast cancer. J Palliat Med 2016;19:863-869.

Address correspondence to: Reina Haque, $P h D, M P H$ Department of Research \& Evaluation Kaiser Permanente Southern California 100 South Los Robles, 2nd Floor Pasadena, CA 91101

USA

E-mail: reina.haque@kp.org 